\title{
El conocimiento florístico de México
}

\author{
PATRICIA DÁVILA ${ }^{1}$ Y VICTORIA SOSA ${ }^{2}$ \\ ${ }^{1}$ Instituto de Biolngía, IJNAM. Apdo. Postal 70-233. 04510 México, D.F. \\ ${ }^{2}$ Instituto de Ecología, A.C. Apdo. Postal 63. 91000, Xalapa, Veracruz
}

\begin{abstract}
Resumen. Se presenta una recapitulación sobre el conocimiento florístico de México con base en el análisis de herbarios, colecciones y estudios florísticos existentes. En la actualidad se estima que existen 2.3 millones de ejemplares de herbario en el país. Se tienen registrados 71 herbarios, de los cuáles la mayoría se concentran en el centro del país. En lo que respecta a los estudios florísticos desarrollados en México, existen tres floras regionales que ya han sido concluídas (Desierto de Sonora, Baja California y Valle de México) y alrededor de 13 más se encuentran en desarrollo. En total, estas 16 floras cubren cerca del $70 \%$ de la superficie de México, aunque la mayoría de las floras en desarrollo requieren aún del estudio de más del $50 \%$ de los taxa distribuidos en sus zonas y aquellas enunciadas como concluidas requieren de una actualización. En lo que respecta a los estudios taxonómicos se estima que un 55\% de las especies vegetales de México ya han sido estudiadas o al menos listadas en algún trabajo. También se señalan algunas perspectivas de los estudios florísticos en México con respecto a la obtención de información de otros herbarios, especialmente del extranjero, para una mejor planificación de las exploraciones botánicas, para la inclusión de nuevas tecnologías, así como para el apoyo a los herbarios y a la formación de recursos humanos.
\end{abstract}

\begin{abstract}
A summary of the floristic knowledge of Mexico, based on the analysis of the existant herbaria, collections and floristic studies is presented. At present it is estimated that there are 2.3 million specimens deposited in Mexican herbaria. There are 71 herbaria in Mexico, most of them located in the central part of the country. There are three floristic studies that had been concluded (Desierto de Sonora, Baja California and Valle de México) and about 13 more are in development. In total, these 16 floras cover about $70 \%$ of the Mexican territory; however, most of the floras in development still need to undertake the study of more than $50 \%$ of the taxa distributed in their respective zones and those that had been concluded need the updating of their information. It is estimated that the taxonomic studies of Mexican taxa covers about $55 \%$ of the plant species of the country. Also, this paper points out some perspectives of the floristic studies in Mexico in relation to the obtention of information from other herbaria especially the foreign ones, to the appropriate planning of botanical explorations, to the inclusion of new technologies, as well as to the herbaria support and their personnel training.
\end{abstract}

\section{INTRODUCCIÓN}

Dentro de este número especial del Boletín de la Sociedad Botónica de México, nos han invitado a recapitular sobre el conocimiento florístico de México con base en una revisión que recientemente presentamos y que fue publicada en extenso (Sosa y Dávila, 1994). A pesar de que algunas secciones e información de este artículo coinciden con el ya publicado, consideramos importante presentarlo dentro del foro de la Sociedad Botánica de México y por lo tanto, agradecemos la oportunidad que se nos brinda de sintetizar algunos aspectos de la información florística en el país, en el marco de este volumen que reúne información sobre las diferentes ramas del quehacer botánico en México.

En este contexto, vale la pena hacer énfasis una vez más en que México es un país con una alta riqueza florística. Se ha estimado que el número de especies fanerogámicas en el país es de airededor de 25,000 (Rzedowski, 1993). Asimismo, se ha calculado que aproximadamente el $10 \%$ de los géneros y el $62 \%$ de las especies son endémicos (Rzedowski, 1993). El mayor número de especies de plantas en México corresponde a las angiospermas y dentro de ellas, las familias más diversas son: Compositae (2,026 especies; Turner y Nesom, 1993), Leguminosae (1,724 especies; Sousa y Delgado, 1993),
Orchidaceae (1,200 especies; Hágsater y Salazar, 1991), Gramineae (1,226 especies; Beetle, 1987a, b), Cactaceae ( 821 especies; Bravo-Hollis, 1978; Bravo-Hollis y Sánchez-Mejorada, 1991a, b) y Rubiaceae (510 especies; Rzedowski, 1993).

También es necesario recalcar la importancia que cobra el conocimiento de la biodiversidad vegetal para la planeación de políticas de conservación, especialmente para resolver problemas de preservación de especies y comunidades en áreas específicas. El papel más importante que pueden jugar los taxónomos en estudios de biodiversidad y conservación es en lo que respecta a la catalogación de esta diversidad, es decir, describirla, identificarla, documentarla e interpretarla en la forma de generalizaciones sobre relaciones históricas, biogeografía y endemismos (Barrowclough, 1992). En otras palabras, estos significa que si los estudios florísticos son puestos en este contexto, estos pueden contribuir grandemente en resolver la llamada crisis de la biodiversidad.

El objetivo del presente trabajo es evaluar el estado actual del conocimiento florístico de México y presentar algunas perspectivas de la investigación en esta área, en el país. Esta evaluación está basada en un análisis del número de colecciones botánicas y de herbarios, así como en los estudios florísticos concluidos y en desarrollo que existen en México. 


\section{COLECCIONES}

Con respecto a las colecciones botánicas, se ha estimado que en la actualidad existen casi 2.3 millones de ejemplares en el país. Su incremento ha sido notable en las últimas décadas, lo cual puede observarse en el Cuadro 1. Igualmente notable resulta el número de ejemplares de plantas mexicanas depositadas en herbarios extranjeros. Rzedowski (1976) estima que este número asciende a 1,800,000 ejemplares.

Cuadro 1. Número de colecciones en México.

\begin{tabular}{lllll}
\hline Año & 1974 & 1981 & 1990 & 1993 \\
\hline $\begin{array}{l}\text { Número de } \\
\text { colecciones }\end{array}$ & 566,780 & $1,021,713$ & $2,107,543$ & $2,284,693$
\end{tabular}

Con base en Index Herbarium: Holmgren y Keuken, 1974, Holmgren et al., 1981, 1990; Consejo Nacional de la Flora de México, inf. inédita.

\section{HERBARIOS}

En relación a los herbarios mexicanos, se sabe que el número actual de los mismos en México asciende a 71 (Sosa y Dávila, 1994). Su incremento ha seguido la misma tendencia que las colecciones (Cuadro 2). La mayoría de los herbarios se concentran en el centro del país. Dentro de ellos están los más grandes y antiguos, como el Herbario Nacional (MEXU) y el Herbario del Instituto Politécnico Nacional (ENCB), y hay otros de origen más o menos reciente. En la región oeste, sólo el Herbario del Instituto de Botánica de la Universidad de Guadalajara (IBUG) alberga más de 100,000 ejemplares. La zona sur posee el menor número de herbarios y colecciones, aunque el tercero más grande del país, el del Instituto de Ecología (XAL), se encuentra localizado en esta área. Hasta el momento, en estados como Zacatecas, Colima, Campeche y Guanajuato, todavía se carece de herbarios.

Cuadro 2. Número de herbarios. en México.

\begin{tabular}{lcccc}
\hline Año & 1974 & 1981 & 1990 & 1993 \\
\hline $\begin{array}{l}\text { Número de } \\
\text { herbarios }\end{array}$ & 18 & 30 & 70 & 71 \\
\hline
\end{tabular}

Con base en Index Herbarium: Holmgren y Keuken, 1974, Holmgren et al., 1981, 1990; Consejo Nacional de la Flora de México, inf. inédita.

\section{ESTUDIOS FLORÍSTICOS}

En lo que respecta a los estudios florísticos desarrollados en México, en los últimos años han sido concluidas tres floras regionales: la del Desierto de Sonora (Wiggins, 1964), la de
Baja California (Wiggins, 1980) y la del Valle de México (Rzedowski y Rzedowski, 1979, 1985, 1990) (Cuadro 3). Trece floras regionales más están en proceso (Cuadro 4). En total, estas 16 floras cubren alrededor del $70 \%$ de la superficie de México. Sin embargo, la mayoría de las floras en desarrollo requiere aun del estudio de más del $50 \%$ de los taxa distribuidos en sus zonas, y es necesaria una actualización para aquellas enunciadas como concluidas.

Cuadro 3.Estudios florísticos concluidos en México.

\begin{tabular}{lcl}
\hline Flora & $\begin{array}{c}\text { Total de especies } \\
\text { conocidas }\end{array}$ & Referencias \\
\hline Baja California & 2,705 & Wiggins, 1980 \\
Desierto Sonorense & 2,634 & Wiggins, 1964 \\
Valle de México & 2,071 & Rzedowskiy Rzedowski, \\
& & $1979,1985,1989,1990$
\end{tabular}

Otra fuente de información florística importante está representada por los estudios taxonómicos de grupos vegetales distribuidos en México (Cuadro 5), y los listados florísticos de áreas geográficas o grupos botánicos (Cuadro 6). Tomando en consideración el cálculo conservador de que en México existen 25,000 especies fanerogámicas, y sobreponiéndole las especies vegetales mexicanas incluidas tanto en floras como monografías y listados, se puede concluir que aproximadamente un 55\% de las mismas ya han sido estudiadas o al menos listadas en algún trabajo.

\section{PERSPECTIVAS}

México ha incrementado notablemente su infraestructura botánica, sus colecciones y su personal entrenado. Esto es notable incluso en comparación con otros países de Latinoamérica (Toledo y Sosa, 1993). Sin embargo, se estima que el $30 \%$ de la superficie del país no está considerada en ningún estudio florístico, además de que existen algunas áreas que todavía no están suficientemente exploradas, botánicamente hablando. Asimismo, un $45 \%$ de las especies estimadas para México no ha sido listado ni estudiado taxonómicamente, y de hecho consideramos que varios grupos de plantas requieren mayor atención, tanto para su recolección, como para su estudio taxonómico.

La información presentada aquí indica que a pesar de que numerosas floras regionales cubren casi tres cuartas partes del país, el tiempo requerido para completarlas es muy grande. Por otro lado, el país no cuenta con una flora a nivel nacional. Por ello se considera que en forma simultánea al programa de colección y al desarrollo de las floras regionales, se requiere la iniciación de una base de datos que permita recuperar fácilmente la información florística disponible. Es innegable que, para lograr esta meta, es indis- 
CuAdro 4. Floras regionales en desarrollo en México.

\begin{tabular}{|c|c|c|c|}
\hline Flora & $\begin{array}{l}\text { Número total } \\
\text { de especies }\end{array}$ & $\begin{array}{l}\text { Especies incluidas } \\
\text { en tratamientos } \\
\text { taxonómicos }\end{array}$ & Referencias \\
\hline Sinaloa & 5000 & $\ldots$ & Vega, 1990 \\
\hline Durango & 4300 & - & González et al., 1990 \\
\hline Nayarit & 4500 & _ & Téllez y Flores, 1990 \\
\hline Novogaliciana & ? & 3523 & McVaugh 1983, 1984, 1985, 1987, 1989, 1992 \\
\hline \multicolumn{4}{|l|}{ Bajío y regiones } \\
\hline adyacentes & .5000 & 77. & Rzedowski y Rzedowski(edrs.) 1991-1993 \\
\hline Veracruz & 7690 & 1059 & Gómez-Pompa (edr.) 1978- 1991; Sosa (edr.) 1992-1993 \\
\hline Tehuacán-Cuicatlán & 3000 & 4 & Dávila et al., (edrs.) 1993 \\
\hline Guerrero & 6500 & 15 & Diego, 1990; López-Ferrari, 1989 \\
\hline Oaxaca & 10000 & & Dávila y Sousa, 1991 \\
\hline Chiapas & 8000 & $\overline{506}$ & Breedlove, 1981; Smith 1981 \\
\hline \multicolumn{4}{|l|}{ Etnoflora } \\
\hline yucatanense & 1936 & 15 & Flores et al., 1992; Trejo y Cian-Uitz, 1992 \\
\hline Jalisco & 7000 & - & Puga, com. pers. \\
\hline Mesoamericana & ? & 3243 & Davidse et al., 1994 \\
\hline
\end{tabular}

Cuadro 5. Estudios taxonómicos de los grupos más diversos distribuidos en México.

\begin{tabular}{|c|c|c|c|}
\hline Grupo de plantas & $\begin{array}{l}\text { Número de } \\
\text { especies }\end{array}$ & $\begin{array}{l}\text { Especies } \\
\text { estudiadas }\end{array}$ & Referencias \\
\hline Cactaceae & 821 & 821 & Bravo-Hollis, 1978; Bravo-Hollis y Sánchez Mejorada, 1991a, \\
\hline Compositae & ca. 2026 & - & Turner y Nesom (en prensa) \\
\hline Gramineae & 1226 & 538 & Beetle, 1983, 1987b, 1991 \\
\hline Malvaceae & 372 & 372 & Fryxell, 1988 \\
\hline Orchidaceae & 1200 & 100 & Hágsater y Salazar (edrs.), 1991 \\
\hline Palmae & 100 & - & Quero (en preparación) \\
\hline Pteridophyta & 690 & 690 & Mickel y Beitel. 1988 \\
\hline
\end{tabular}

Cuadro 6. Listados florísticos de México.

Área geográfica o

grupo de plantas
Número reportado

de especies

\begin{tabular}{ll}
\hline Chiapas & 7018 \\
Cozumel & 542 \\
Durango & 3800 \\
Estación de Biología Chamela & 754 \\
Estación de Biología Los Tuxtlas & 818 \\
Península de Yucatán & 1936 \\
Querétaro & 2334 \\
Quintana Roo & 1300 \\
Tabasco & 2147 \\
Tehuacán-Cuicatlán & 2700 \\
Angiospermas acuáticas & 111 \\
Monocotiledóneas Parte 1 & 393 \\
Gramíneas de Puebla & 427 \\
Pteridofitas de Gunajuato, & 300
\end{tabular}

Referencias

Breedlove, 1986

Téllez y Cabrera, 1987

González et al., 1991

Lott, 1985

Ibarra y Sinaca, 1987

Sosa et al., 1985

Argüelles et al., 1991

Sousa y Cabrera, 1983

Cowan, 1983

Dávila et al., 1993

Lot et al., 1986

Espejo y López-Ferrari, 1992

Dávila et al., 1990

Díaz y Palacios-Ríos, 1992 
pensable la cooperación de todos los botánicos y herbarios mexicanos, así como de los taxónomos extranjeros interesados en la flora del país. Se ha reconocido ampliamente la importancia de que la información que se tiene sobre diversidad vegetal sea de fácil acceso, tanto para el público en general como para aquellas personas responsables de tomar decisiones en el campo de la conservación de los recursos (Morin y Gomon, 1993; Systematics Agenda 2000, 1994). Por lo tanto, es necesario conjuntar esta información en un tiempo razonablemente corto, sin perder con ello la calidad y precisión de la misma.

A pesar de que en México se notan avances sustanciales en lo que corresponde al conocimiento de su flora, la información que presentamos indica que aun es necesario desarrollar programas intensivos de colecta en áreas del país poco conocidas florísticaments. Se requieren colectas intensivas en ciertas áreas, entre las que podemos mencionar a las selvas tropicales en Chiapas, Guerrero y Oaxaca y las zonas desérticas del norte del país. A nivel estatal, prácticamente no existe información florística de Zacatecas, Colima, Campeche y Guanajuato. Ahora bien, con base en la información presentada por Rzedowski (1991), vale la pena llevar a cabo dentro de estas grandes áreas, estudios florísticos en zonas específicas en donde las comunidades vegetales involucradas son portadoras de una mayor riqueza florística y un mayor número de endemismos. Desde el punto de vista de su riqueza florística, es importante continuar las exploraciones botánicas en los bosques mesófilos de montaña, de coníferas y encinos, en los matorrales xerófilos y en los bosques tropicales caducifolios y subcaducifolios principalmente. Desde el punto de vista del endemismo, resulta prioritario llevar a cabo estudios florísticos en los matorrales xerófilos y en bosques mesófilos de montaña.

Se han señalado hasta el momento datos y cifras con respecto a herbarios, colecciones y proyectos florísticos. En consecuencia, debemos tratar de integrarlos en el panorama actual del conocimiento florístico nacional y de sus perspectivas dentro del contexto de las necesidades y problemática de México. Si bien es cierto que algunas floras están en una etapa muy àctiva o bien ya han sido concluidas, aun no existe una flora sintética del país. Sin embargo, con todos los datos que se mostraron anteriormente creemos que ya existe un buen cúmulo de información sobre la flora mexicana, que vale la pena conjuntar y publicar, como un primer paso encaminado a la elaboración de la flora de México.

Si nuestras estimaciones son correctas, tenemos que aproximadamente el $70 \%$ de la superficie del país está florísticamente conocido o próximo a serlo, con base en los proyectos en desarrollo. Por otro lado, se considera que del $30 \%$ restante del territorio, la flora puede ser parcialmente reconocida apoyándose en el conocimiento florístico existente en las regiones adyacentes. Por ejemplo, la flora del estado de Colima puede reconocerse en gran medida gracias a la información existente para los estados de Jalisco,
Michoacán, Durango y Nayarit. Para el estado de Zacatecas, la información ya existente de los estados de Durango, Jalisco y Nayarit es de gran valía. En el caso del estado de Tlaxcala, la información florística que existe para los estados de México, Veracruz y parcialmente para el estado de Puebla, nos brinda en conjunto la oportunidad de reconocer parte de su acervo florístico. Esto no implica, por supuesto, que no se requieran programas serios y planificados de recolección botánica en estas entidades, sino que parte de su flora puede ser reconocida por el conocimiento existente en zonas adyacentes, florísticamente parecidas.

Asimismo, es importante que los estudios florísticos incorporen nuevas tecnologías, como sería la formación de bancos de datos ligados a sistemas de información geográfica y ecológica. Esto permite obtener y manejar información básica e importante para cualquier estudio involucrado en la protección, conservación y manejo de recursos vegetales.

Hay muchas necesidades que el ámbito botánico debe resolver para llegar a conjuntar adecuadamente la Flora de México, la que necesariamente debe formularse con base en un buen programa de floras regionales, flórulas de zonas clave ya sea por su diversidad y/o endemismo, o bien de grupos taxonómicos importantes por su diversidad, endemismo, uso o algún aspecto ecológico sobresaliente.

Respecto a las necesidades que requieren los proyectos florísticos, a continuación presentamos y discutimos algunas de ellas.

\section{FORTALECIMIENTO Y APOYO A LA INFRAESTRUCTURA DE HERBARIOS Y COLECCIONES}

Es común que en algunas instituciones científicas y en otras que brindan apoyo económico para realizar los proyectos de investigación, no entiendan las grandes necesidades que todos los herbarios tienen. La dificultad para contar con edificios adecuados, y equipo y mobiliario en los herbarios, sigue siendo un cuello de botella que impide el crecimiento y desarrollo de los mismos. Esta falta de comprensión y credibilidad también se ve reflejada en los proyectos de investigación que se desarrollan al seno de los herbarios. Es importante que los botánicos y las instituciones que financian proyectos en esta área, comprendan que como cualquier otra rama científica, los herbarios y los que en ellos trabajamos requerimos infraestructura adecuada y apoyo financiero para el desarrollo de nuestros proyectos.

En este mismo sentido, también se considera adecuado que el medio científico y las agencias que financian los proyectos de investigación, tengan claro que las colecciones biológicas son herramientas necesarias para la investigación, por lo cual deben de recibir el mismo apoyo y reconocimiento que otras bases de datos sobre diversidad biológica (Alberch, 1993) o como los laboratorios donde se llevan a cabo estudios experimentales sofisticados. Es claro que las colecciones biológicas no representan un fin por sí mismas, 
sino que forman parte de la investigación sistemática de cualquier botánico.

Un problema generalizado, y que no atañe únicamente a México, es el hecho de que en las colecciones biológicas: 1) la información no ha sido sistematizada en bases de datos, con el fin de hacerla accesible a la comunidad; 2) las bases de datos existentes no han sido organizadas bajo los mismos criterios, y 3 ) las bases de datos no comparten los mismos estándares de información (Alberch, 1993). Por lo tanto, dentro de las alternativas para fortalecer los herbarios mexicanos será necesario tomar en cuenta este problema que día con día adquiere mayor relevancia, no sòlo en el ámbito científico, sino en aspectos relacionados con estudios de ordenamiento ecológico y conservación y manejo de recursos bióticos.

\section{APOYO PARA LLEVAR A CABO EXPLORACIONES BOTÁNICAS}

Es imprescindible apoyar las exploraciones botánicas en el país, siempre y cuando éstas se lleven a cabo de una manera organizada y planificada, dando preferencia a la recolección de material en peligro de desaparecer, con alto número de endemismos y de especies, y en zonas poco exploradas. Aquí nuevamente se requiere de un apoyo financiero sostenido para llevar a cabo la recolección de material botánico en forma sistemática y continua. Es innegable que el proceso comprendido entre el desarrollo del trabajo de campo, el trabajo de herbario y su culminación a través de un trabajo florístico o sistemático es en ocasiones lento, pero insustituible y de gran importancia. Por lo tanto estas necesidades y tiempos deben quedar estipulados por el proponente del proyecto y comprendido por quien otorga el financiamiento. En esta actividad es recomendable una coordinación entre los diferentes proyectos florísticos que se inician, considerando tanto las áreas de recolección, como los grupos de plantas involucrados.

\section{PERSONAL CAPACITADO}

En este punto invariablemente llegamos a un tema importante que ya ha sido discutido en otros foros científicos. Aunque ya se están formando taxónomos en diferentes instituciones del país, lo cual vemos con optimismo, México carece aun de un posgrado especializado en taxonomía. Esta problemática es extremadamente compleja y solamente nos atrevemos a exponer algunas ideas que ponemos a la consideración de todos los botánicos mexicanos y de aquellos interesados en el quehacer taxonómico del país. En la actualidad, existe en el ámbito científico una revaloración del trabajo taxonómico, incluyendo el florístico. Además, existe una masa crítica de taxónomos de carrera de buen nivel, que en conjunto podrían formar una planilla de profesores del posgrado en taxonomía. Este grupo de profesores, utilizando su experiencia y conocimientos, estarían capacitados para proponer un plan de estudios moderno y que cumpla con las necesidades del país. Por otra parte, existen varias instituciones en México que podrían albergar y administrar en sus instalaciones el posgrado en taxonomía, en donde jóvenes interesados en incorporarse a un posgrado en taxonomía, y que no han encontrado un lugar adecuado para cumplir sus objetivos, puedan recibir una formación adecuada. Finalmente, los estudios en taxonomía y florística tienen una incidencia en aspectos de conservación y manejo de recursos genéticos.

A pesar de esto, no ha surgido una institución ni un grupo de trabajo interesado en iniciar una empresa tan importante para el país, como lo es la organización de un Posgrado en Taxonomía. Es recomendable que este posgrado surja pronto, como una propuesta organizada y coordinada de taxónomos e instituciones del país. De no hacerlo en conjunto, se corre el riesgo de empezar a formar posgrados en diferentes instituciones que tengan programas similares o idénticos, lo que diluiría el esfuerzo y el buen nivel que podría alcanzarse al trabajar en forma coordinada.

La integración de un programa de posgrado en taxonomía como el que se sugiere, tiene que hacer frente a las necesidades más apremiantes en México, como son las relacionadas con los inventarios biológicos, pero sin descuidar otros aspectos del quehacer taxonómico. Existe en la actualidad una controversia sobre la distracción que pueden representar los inventarios florísticos y faunísticos en el desarrollo teórico de la sistemática (Renner y Ricklefs, 1994). Por ello, para atraer estudiantes, este programa de posgrado tendrá que hacer énfasis en que la taxonomía es una ciencia que busca respuestas a preguntas biológicas fundamentales. Esto se ha intensificado con el desarrollo de análisis formales de la información, como la fenética o la cladística, y con el desarrollo de metodologías experimentales que nos permiten capturar, evaluar, integrar e interpretar la información proveniente del ADN. Igualmente, un posgrado como el que proponemos deberá resaltar la importancia de la realización de monografías de grupos taxonómicos, que son un producto muy importante de la investigación taxonómica y parte fundamental de todas las otras investigaciones relacionadas con la historia natural de los grupos (Kellog, 1994).

En otras palabras, en un país como México, con tan alta diversidad vegetal, los estudios florísticos pueden llegar a cobrar gran importancia. Sin embargo, hay que hacer notar que estos estudios son sólo uno de los productos taxonómicos. La actividad taxonómica implica un proceso circular, en donde la exploración, los inventarios, las floras, las monografías, los estudios filogenéticos y las bases de datos son etapas de un proceso (Systematics Agenda 2000, 1994). Para llevar a cabo un estudio de cualquier grupo de organismos, los taxónomos siguen los métodos de explorar, preparar colecciones, inventariar, descubrir relaciones filogenéticas del grupo y elaborar floras y monografías. Esto significa que la actividad taxonómica no es un proceso lineal, y que no es necesario concluir el inventario de una zona o de un país para continuar a nivel experimental o biosistemático hasta llegar a un análisis de integración, como se ha planteado (Chiang, 
1994). Por ello es necesario enfatizar que es igualmente importante dar apoyo a todas las actividades taxonómicas y que cada una de las fases de la taxonomía es igualmente importante.

El papel que pueden jugar los taxónomos en la crisis actual de la biodiversidad, en especial en México, puede ser muy valioso si se apoya y estimula cada una de las actividades taxonómicas. Considerando dentro de este contexto a la información florística, y proporcionándola en forma accesible al resto de la comunidad científica y a los responsables de tomar decisiones en materia de biodiversidad, se facilitará en gran medida la planeación de políticas sobre conservación.

\section{LITERATURA CITADA}

Alberch P. 1993. Museums, collections and biodiversity inventories. TREE 8: 372-375.

Argüelles E., Fernández R., Zamudio S. 1991. Listado preliminar del estado de Querétaro. En: Rzedowski J, Rzedowski GC de, edrs. Flora del Bajịo y regiones adyacentes. Fasc. Comp. 2. Instituto de Ecología, A.C. Pátzcuaro, México, 1-155.

Barrowclough GF.1992. The meaning of systematics and the biodiversity crisis. En: Eldredge N, edr. Systematics, ecology and the biodiversity crisis. Columbia University Press, New York, 121-124.

Beetle AA. 1983. Las gramíneas de México. Vol. 1. Secretaría de Agricultura y Recursos Hidraúlicos, México,

Beetle AA. 1987a. Noteworthy grassess from Mexico XIII. Phytologia 63: 209-297.

Beetle AA 1987b. Las gramíneas de México. Vol. 2. Secretaría de Agricultura y Recursos Hidráulicos, México.

Beetle AA. 1991. Las gramíneas de México. Vol. 3. Secretaría de Agricultura y Recursos Hidráulicos, México.

Bravo-Hollis H. 1978. Las cactáceas de México. Vol. 1. UNAM, México.

Bravo-Hollis H, Sánchez-Mejorada 1991a. Las cactáceas de México. Vol.2. UNAM, México.

Bravo-Hollis H, Sánchez-Mejorada 1991b. Las cactáceas de México. Vol.3. UNAM, México.

Breedlove DE. 1981. Introduction to the flora of Chiapas. Part 1. California Academy of Sciences, San Fco.

Breedlove DE. 1986. Listados florísticos de México. IV. Flora de Chiapas. Instituto de Biología, UNAM, México.

Chiang F. 1994. La taxonomía vegetal en México: problemas y perspectivas. En: Llorente J, Luna I, edrs. Taxonomía biológica. Fondo de Cultura Económica, México, 291-297.

Cowan CP. 1983. Listados florísticos de México. I. Flora de Tabasco. Instituto de Biología, UNAM, México.

Davidse G, Sousa M, Chater AO, edrs. 1994. Flora Mesoamericana, Vol. 6, Alismataceae a Cyperaceae. UNAM, México; Missouri Botanical Garden, St. Louis; The Natural History Museum, London.

Dávila P, Sousa M. 1991. Flora de Oaxaca. Guía de autores e introducción sobre aspectos físico-ambientales y vegetación. Instituto de Biología, UNAM, México.

Dávila P., P. Tenorio P, Manrique E, Miranda A, Rodríguez A. 1990. Listados florísticos de México. VIII. Listado florístico de las gramíneas de Puebla. Instituto de Biología, UNAM, México.

Dávila P, Villaseñor JL, Medina R, Ramírez A, Salinas A, SánchezKen J, Tenorio P. 1993. Listados florísticos de México X. Flora del Valle de Tehuacán-Cuicatlán. Instituto de Biología,
UNAM, México .

Dávila P, Villaseñor JL, Medina R, Téllez O, edrs. 1993. Flora del Valle de Tehuacán-Cuicatlán. Fascs. 1-7. Instituto de Biología, UNAM, México.

Díaz H, Palacio-Ríos M. 1992. Listado preliminar de especies de pteridofitas de los estados de Guanajuato, Michoacán y Querétaro. En: Rzedowski J, y Rzedowski GC de, edrs. Flora del Bajio y regiones adyacentes. Fasc. Comp. 3. Instituto de Ecología, A.C., Pátzcuaro, México, 1-57

Diego N. 1990. Flora del estado de Guerrero. En: Resúmenes del XI Congreso Mexicano de Botánica, Sociedad Botánica de México, México.

Espejo A, López-Ferrari A. 1992. Las monocotiledóneas mexicanas: Una sinopsis florística. 1. Lista de Referencia. Vol.1. Consejo Nacional de la Flora de México-UAM Iztapalapa, México.

Flores JS, Narave H, Vovides AP. 1992. Gymnospermae. En: Flores JS, edr. Etnoflora Yucatanense. Fasc. 5. Universidad Autónoma de Yucatán, Mérida, México.

Fryxell PA. 1988. Malvaceae of Mexico. Syst. Bot. Monogr. 25: 1-522

Gómez-Pompa A,edr.1978-1991. Flora de Veracruz. Fasc. 1-66. INIREBInstituto de Ecología A.C., Xalapa, Veracruz, México.

González S, González ME, Arrieta H. 1991. Listados florísticos de México IX. Flora de Durango. Instituto de Biología, UNAM, México.

González S, González ME, García A. 1990. La flora de Durango. En: Resúmenes del XI Congreso Mexicano de Botánica, Sociedad Botánica de México. México.

Hágster E, Salazar G, edrs. 1991. Orquídeas dẻ México. Icones Orchidacearum. Vol.1. Asociación Mexicana de Orquideología México.

Holmgren PK, Keuken W. 1974. Index Herbariorum. Part 1. The herbaria of the world. 6th ed. Regnum Veg. 92.

Holmgren PK, Keuken W, Schofield EK. 1981. Index herbariorum. Part 1. The herbaria of the world. 7th ed. Regnum Veg. 106.

Holmgren PK, Holmgren NH, Barnett L. 1990. Index herbariorum. Part 1. The herbaria of the world. 8th ed. Regnum Veg. 120.

Ibarra G, Sinaca S. 1987. Listados florísticos de México VII. Estación de Biología Tropical Los Tuxtlas, Veracruz. Instituto de Biología, UNAM, México.

Kellog EA. 1994. What makes a good monograph? ASPT Newsletter 8: $12-15$.

López-Ferrari AR. 1989. Araliaceae. En: Diego N, edra. Flora de Guerrero. Vol. 1. Facultad de Ciencias, UNAM, México, 1-23

Lot A, Novelo A, Ramírez-Mercado P. 1986. Listados florísticos de México. V. Angiospermas acuáticas mexicanas 1 . Instituto de Biología, UNAM, México.

Lott EJ. 1985. Listados florísticos de México. III. La Estación de Biología Chamela, Jalisco. Instituto de Biología, UNAM, México.

McVaugh R. 1983. Gramineae. En: Anderson WR, edr. Flora Novogaliciana. Vol. 14, Universidad Michigan Press, Ann Arbor.

McVaugh R. 1984. Compositae. En: Anderson WR, edr. Flora Novogaliciana. Vol. 12, Universidad Michigan Press, Ann Arbor.

McVaugh R. 1985. Orchidaceae. En: Anderson WR, edr. Flora Novogaliciana. Vol. 16, Universidad Michigan Press, Ann Arbor.

McVaugh R. 1987. Leguminosae. En: Anderson WR, edr. Flora Novogaliciana. Vol. 5, Universidad Michigan Press, Ann Arbor.

McVaugh R. 1989. Bromeliaceae to Dioscoreaceae. En: Anderson WR, edr. Flora Novogaliciana. Vol. 15, Universidad Michigan Press, Ann Arbor. 
McVaugh R. 1992. Gymnosperms and Pteridophytes. En: W. R. Anderson WR, edr. Flora Novogaliciana Vol. 17, Universidad Michigan Press, Ann Arbor.

Mickel JT, Beittel JM. 1988. Pteridophyte Flora of Oaxaca, Mexico. Mem. New York Bot. Gard. 46: 1-568.

Morin NR, Gomon J. 1993. Data banking and the role of natural history collections. Ann. Missouri Bot. Gard. 80: 317-322.

Renner SS, Ricklefs RE. 1994. Systematics and biodiversity. TREE 9: 78.

Rzedowski J. 1976. Catálogo de los herbarios institucionales mexicanos. Sociedad Botánica de México, México.

Rzedowski J. 1991. El endemismo de la flora fanerogámica mexicana: Una apreciación analítica preliminar. Acta Bot. Mex. 15: 47-64.

Rzedowski J. 1993. Diversity and origins of the phanerogamic flora of Mexico. En: Ramamoorthy TP, Bye R, Lot A, Fa J, edrs. Biological diversity of Mexico: Origins and distribution. Oxford Universidad Press, New York, 129-148.

Rzedowski J, Rzedowski GC de. 1979. Flora fanerogámica del Valle de México. Vol. 1. Compañía Editorial Continental, México.

Rzedowski J, Rzedowski GC de. 1985. Flora fanerogámica del Valle de México. Vol. 2. Escuela Nacional de Ciencias BiólogicasInstituto de Ecología, A.C. México.

Rzedowski J, Rzedowski GC de. 1989. Sinopsis numérica de la flora fanerogámica del Valle de México. Acta Bot. Mex. 8: 15-30.

Rzedowski J, Rzedowski GC de. 1990. Flora fanerogámica del Valle de México. Vol. 3. Instituto de Ecología, Pátzcuaro, México.

Rzedowski J, Rzedowski GC, edrs. 1991-1993. Flora del Bajío y regiones adyacentes. Fasc. 1-16. Instituto de Ecología, A.C. Pátzcuaro, México.

Smith AR. 1981. Pteridophytes. En: Breedlove DE, edr. Flora of Chiapas. Proc. Calif. Acad. Sci. 2: 1-370.

Sosa V. edra. 1992-1993. Flora de Veracruz. Fasc. 67-72. Instituto de Ecología, A.C., Xalapa, Veracruz, México.

Sosa V, Dávila P. 1994. Una evaluación del conocimiento florístico de México. Ann. Missouri Bot. Gard. 81: 749-757.
Sosa V, Flores JS, Rico-Garay V, Lira R, Ortiz JJ. 1985. Lista florística y sinonimia Maya. En: Sosa V, edra. Etnoflora Yucatanense. Vol.1. Instituto Nacional de Investigaciones sobre Recursos Bióticos, Xalapa, México.

Sousa M, Cabrera EF. 1983. Listados florísticos de México II. Flora de Quintana Roo. Instituto de Biología, UNAM, México.

Sousa M, Delgado AS. 1993. Mexican Leguminosae: phytogeography, endemism, and origins. En: Ramamoorthy TP, Bye R, Lot A, Fa J, edrs. Biological diversity of Mexico: Origins and distribution. Oxford Universidad Press, New York, 459-511.

Systematics Agenda 2000.1994. Technical Report. American Museum of Natural History and New York Botanical Garden, 459-511.

Téllez O, Cabrera EC. 1987. Listados florísticos de México VI. Flórula de la Isla de Cozumel, Quintana Roo. Instituto de Biología, UNAM, México.

Tellez O, Flores A. 1990. La flora de Nayarit. En: Resúmenes del XI Congreso Mexicano de Botánica. Sociedad Botánica de México, México, 494.

Toledo VM, Sosa V. 1993. Floristics in LatinAmerica and the Caribbean: An evaluation of the number of plant collections and botanists. Taxon 42: 355-364.

TrejoJC, Ciau-Uitz R. 1992. Psilotaceae. En: Flores JS, edr. Etnoflora Yucatanense. Vol.6. Universidad Autónoma de Yucatán, Mérida, México.

Turner BL, Nesom GL. 1993. Biogeography, diversity and endangered or threatened status of Mexican Asteraceae. En: Ramamoorthy TP, Bye R, Lot A, Fa J, edrs. Biological diversity of Mexico: Origins and distribution. Oxford Univ. Press, New York, 559-576.

Vega R. 1990. Flora de Sinaloa. En: Resúmenes del XI Congreso Mexicano de Botánica. Sociedad Botánica de México, México, 366.

Wiggins IL. 1964. Flora of the Sonoran Desert. En: Shreve F, Wiggins IL, edrs. Vegetation and flora of the Sonoran Desert. Vols. 1, 2, Stanford Univ. Press, California.

Wiggins IL. 1980. Flora of Baja California. Stanford Univ. Press, California. 